Document downloaded from:

http://hdl.handle.net/10251/120362

This paper must be cited as:

Burgos-Simon, C.; Calatayud-Gregori, J.; Cortés, J.; Villafuerte, L. (2018). Solving a class of random non-autonomous linear fractional differential equations by means of a generalized mean square convergent power series. Applied Mathematics Letters. 78:95-104. https://doi.org/10.1016/j.aml.2017.11.009

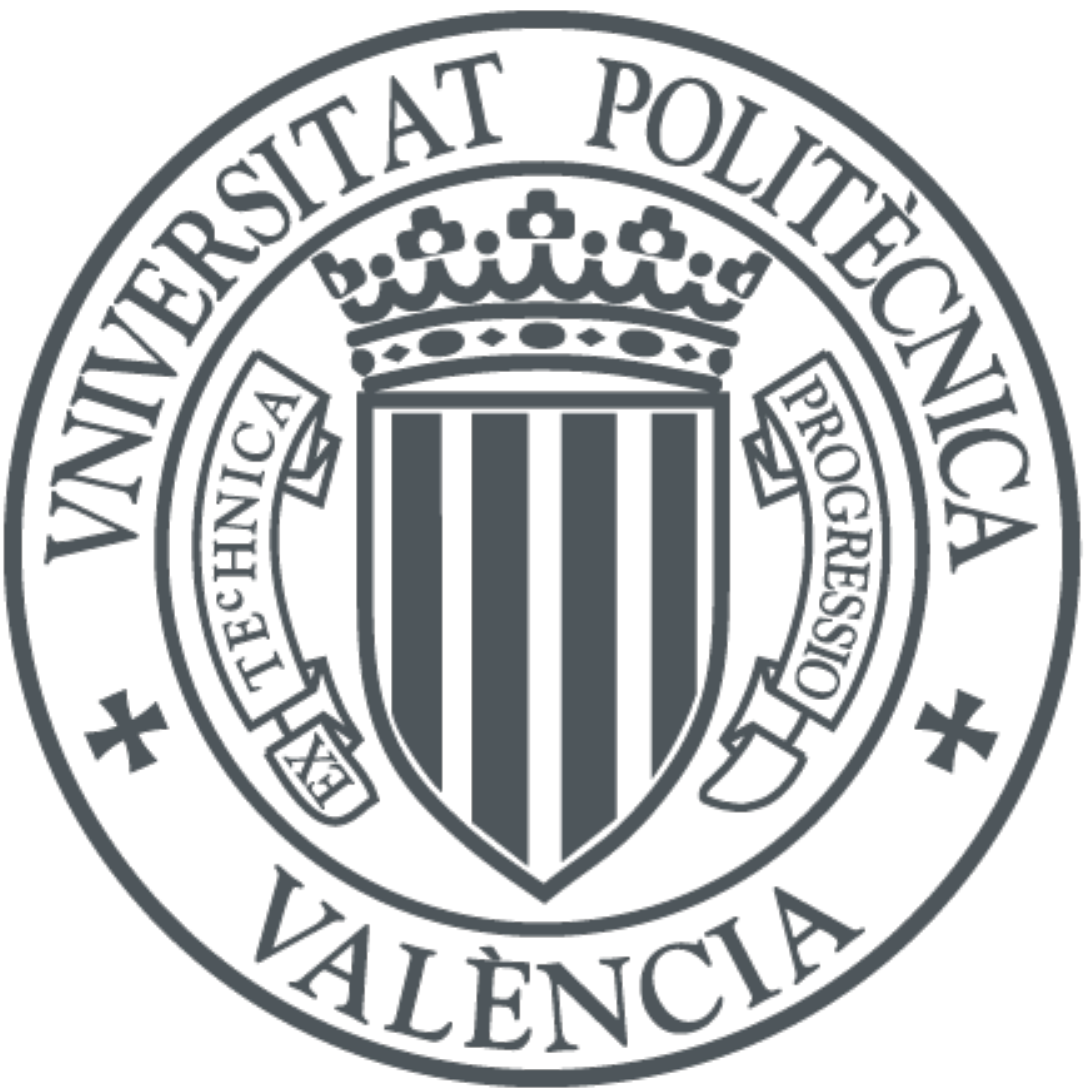

The final publication is available at

http://doi.org/10.1016/j.aml.2017.11.009

Copyright Elsevier

Additional Information 


\title{
Solving a class of random non-autonomous linear fractional differential equations by means of a generalized mean square convergent power series
}

\author{
C. Burgos ${ }^{\mathrm{a}}$, J. Calatayud ${ }^{\mathrm{a}}$, J.-C. Cortés ${ }^{\mathrm{a}, *}$, L. Villafuerte ${ }^{\mathrm{b}}$ \\ a Instituto Universitario de Matemática Multidisciplinar, \\ Universitat Politècnica de València, \\ Camino de Vera s/n, 46022, Valencia, Spain \\ ${ }^{b}$ Department of Mathematics, \\ University of Texas at Austin, USA
}

\begin{abstract}
The aim of this paper is to solve a class of non-autonomous linear fractional differential equations with random inputs. A mean square convergent series solution is constructed in the case that the fractional order $\alpha$ of that Caputo derivative lies in $] 0,1]$ using a random Fröbenius approach. The analysis is conducted by using the so-called mean square random calculus. The mean square convergence of the series solution is established assuming mild conditions on random inputs (diffusion coefficient and initial condition). We show that these conditions are satisfied for a variety of unbounded random variables. In addition, explicit expressions to approximate the mean, the variance and the covariance functions of the random series solution are given. Two full illustrative examples are shown.
\end{abstract}

Keywords: Random fractional differential equations, random mean square calculus.

\section{Motivation and preliminaries}

The combination of random/stochastic and fractional calculus is gaining influence in applied mathematics over the last few years through stochastic/random fractional differential equations (SFDEs/RFDEs). On the one hand, fractional calculus provides a powerful generalization of the classical derivative which is able to model memory and hereditary properties of various materials and processes, like viscoelasticity, phenomena with microscopic complex behaviour (fractals), etc., [? ? ? ? ? ]. On the other hand, stochastic/random calculus is the natural framework to describe phenomena with inherent uncertainty usually meet in physics, biology, engineering, finance, etc. There are two main approaches when uncertainty is considered in fractional differential equations, namely, SFDEs and RFDEs. In the former case, uncertainty is usually modelled through a stochastic process, like Wiener process, having an irregular (e.g., continuous but nowhere differentiable) sample behaviour [? ]. In this approach uncertainty is often restricted to specific probabilistic patterns (typically Gaussian, Poisson, Markovian, etc.). RFDEs are those in which random effects are directly manifested in input parameters (initial/boundary conditions, source terms, coefficients, etc.), which seems to be more natural, since in many models they have a physical interpretation susceptible to encapsulate some kind of uncertainty due to measurement errors and/or the inherent complexity of the phenomenon under analysis [? ]. Another important advantage of RFDEs is that inputs can have a wide variety of probability distributions like Binomial, Poisson, Beta, Gamma, Gaussian, etc. In the extant literature, most of the contributions have focussed on SFDEs. Some recent contributions dealing with existence and uniqueness to solutions of RFDEs can be found in [? ? ]. These results extend their deterministic counterpart. The goal of this paper is to contribute to the emergent area of RFDEs by randomizing a class of non-autonomous fractional differential equations (see (1)) that has been studied, in its deterministic formulation, using the successive approximation method or Picard's method (see, [? , p.232]). As

\footnotetext{
* Corresponding author

Email addresses: clabursi@posgrado.upv.es (C. Burgos), jucagre@alumni.uv.es (J. Calatayud), jccortes@imm.upv.es (J.-C. Cortés), lva5@hotmail. com (L. Villafuerte)
} 
we shall see later, we deal with its random formulation and will construct its solution by applying a random Fröbenius method assuming mild conditions upon random input data.

Let us consider the following random non-autonomous fractional initial value problem (IVP)

$$
\left\{\begin{array}{cl}
\left({ }^{C} D_{0^{+}}^{\alpha} Y\right)(t)-B t^{\beta} Y(t) & =0, \quad t>0, \quad 0<\alpha \leq 1, \quad \beta>0, \\
Y(0) & =A,
\end{array}\right.
$$

where $\left({ }^{C} D_{0^{+}}^{\alpha} Y\right)(t):=\frac{1}{\Gamma(1-\alpha)} \int_{0}^{t}(t-u)^{-\alpha} Y^{\prime}(u) \mathrm{d} u, 0<\alpha \leq 1$, is the random mean square (m.s.) Caputo fractional derivative of order $\alpha$ of the stochastic process $Y(t)$, see [?] and references therein. The input data $A$ and $B$ are assumed to be independent real random variables (RVs) defined in the Hilbert space $\left(\mathrm{L}^{2}(\Omega),\|\cdot\|_{2}\right)$ of second order RVs (2-RVs)

$$
\mathrm{L}^{2}(\Omega)=\left\{X: \Omega \longrightarrow \mathbb{R}:\left(\mathbb{E}\left[X^{2}\right]\right)^{1 / 2}<+\infty\right\}, \quad\|X\|_{2}=\left(\mathbb{E}\left[X^{2}\right]\right)^{1 / 2},
$$

where $\mathbb{E}[\cdot]$ stands for the expectation operator and $(\Omega, \mathcal{F}, \mathbb{P})$ denotes the underlying complete probability space for $A$ and $B$. The norm $\|\cdot\|_{2}$, usually referred to as 2-norm, is inferred from the inner product $\langle X, Y\rangle=\mathbb{E}[X Y], X, Y \in \mathrm{L}^{2}(\Omega)$. Notice that every RV with finite variance belongs to $\mathrm{L}^{2}(\Omega)$. This class of RVs is met in the most part of physical problems involving randomness. Given $\mathcal{T} \subset \mathbb{R}$, if $Z(t) \equiv\{Z(t): t \in \mathcal{T}\}$ is a $2-\mathrm{RV}$ for every $t \in \mathcal{T}$, then $Z(t)$ is termed a second-order stochastic process (2-SP). The convergence inferred by the 2-norm is referred to as mean square (m.s.) convergence. Unless otherwise indicated, throughout this paper we will consider 2-RVs and 2-SPs.

The aim of the paper is to find general conditions on 2-RVs $A, B$ so that for the random IVP (1) we can construct a m.s. solution of the form

$$
Y(t)=\sum_{m=0}^{\infty} X_{m} t^{(\alpha+\beta) m}
$$

where $\left\{X_{m}: m \geq 0\right\}$ is a sequence of $2-\mathrm{RV}$ s to be determined. The study will be conducted by using the random m.s. calculus, see [?]. We recall that a 2-SP $\{Z(t): t \in \mathcal{T}\}$ is m.s. differentiable at $t_{0} \in \mathcal{T}$ with m.s. derivative $Z^{\prime}\left(t_{0}\right)$ if $\lim _{h \rightarrow 0}\left\|\frac{Z\left(t_{0}+h\right)-Z\left(t_{0}\right)}{h}-Z^{\prime}\left(t_{0}\right)\right\|_{2}=0$. The next result provides information of the m.s. square derivative of the product of a deterministic function with a stochastic process.

Theorem 1. [? ] If $f$ is deterministic differentiable at $t_{0}$ and the 2-SP $Z(t)$ is m.s. differentiable at $t_{0}$, then the 2-SP $U(t)=f(t) Z(t)$ is m.s. differentiable at $t_{0}$ and its m.s. derivative is given by $U^{\prime}(t)=f\left(t_{0}\right) Z^{\prime}\left(t_{0}\right)+f^{\prime}\left(t_{0}\right) Z\left(t_{0}\right)$.

Finally, we state a result for differentiating random series in the mean square sense that will be needed later.

Theorem 2. [? , p. 1260] Assume that for $m \geq m_{0} \geq 0, m_{0}$ integer, the process $\left\{V_{m}(u): u \in I\right\}$ satisfies

i) $V_{m}(u)$ is m.s. differentiable on I and $V_{m}^{\prime}(u)$ is m.s. continuous on I,

ii) $V(u)=\sum_{n \geq m_{0}} V_{n}(u)$ is m.s. convergent on $I$,

iii) $\sum_{n \geq m_{0}} V_{m}^{\prime}(u)$ is m.s. uniformly convergent in a neighborhood of each $u \in I$.

Then, for each $u \in I, V(u)$ is m.s. differentiable and $V^{\prime}(u)=\sum_{n \geq 1} V_{n}^{\prime}(u)$.

\section{Constructing a mean square convergent random generalized power series solution and approximating its main statistical properties}

First, we shall justify that the first m.s. derivative of the 2-SP $Y(u)$ defined in (3) at $t=u>0$ is given by

$$
Y^{\prime}(u)=\sum_{m=0}^{\infty} X_{m}(\alpha+\beta) m u^{(\alpha+\beta) m-1} .
$$

To this end, we apply Theorems 1 and 2. Let $u_{0}>0$ be fixed and define $V_{m}(u)=X_{m} u^{(\alpha+\beta) m}$. Let us assume that $X_{m}$ is a 2-RV. By applying Thm. 1, with $f(u)=u^{(\alpha+\beta) m}$ and $Z(u)=X_{m}$, it follows that for each $m, V_{m}(u)$ is m.s differentiable at $u=u_{0}$ and its m.s. derivative is given by $V_{m}^{\prime}\left(u_{0}\right)=X_{m}(\alpha+\beta) m u_{0}^{(\alpha+\beta) m-1}$. It is easy to check that $V_{m}(u)$ is m.s. continuous at $u_{0}$. Once coefficients $X_{m}$ are determined, we will find conditions on $\mathrm{RVs} A, B$ in order 
to hypotheses ii) and iii) of Thm. 2 are met. For now on, assume that $I \subset[0, \infty)$. If $V(u)=\sum_{n \geq m_{0}} V_{n}(u)$ is m.s. convergent on $I$ and $\sum_{n \geq m_{0}} V_{m}^{\prime}(u)$ is m.s. uniformly (m.s.u.) convergent in a neighbourhood of each $u \in I$, then Thm. 2 implies (4) and

$$
\begin{aligned}
\left({ }^{C} D_{0^{+}}^{\alpha} Y\right)(t) & =\frac{1}{\Gamma(1-\alpha)} \int_{0}^{t}(t-u)^{-\alpha} Y^{\prime}(u) \mathrm{d} u \\
& =\frac{1}{\Gamma(1-\alpha)} \int_{0}^{t}(t-u)^{-\alpha} \sum_{m=0}^{\infty} X_{m}(\alpha+\beta) m u^{(\alpha+\beta) m-1} \mathrm{~d} u \\
& =\sum_{m=0}^{\infty} X_{m}(\alpha+\beta) m \frac{1}{\Gamma(1-\alpha)} \int_{0}^{t}(t-u)^{-\alpha} u^{(\alpha+\beta) m-1} \mathrm{~d} u
\end{aligned}
$$

The commutation of the series in the last step is legitimated because it is m.s.u. convergent. Now, using the substitution $u=v t$ on the above integral and the relationship between beta, $\operatorname{Be}(\cdot, \cdot)$, and gamma, $\Gamma(\cdot)$, special functions, namely, $\Gamma\left(\alpha_{1}\right) \Gamma\left(\alpha_{2}\right) / \Gamma\left(\alpha_{1}+\alpha_{2}\right)=\operatorname{Be}\left(\alpha_{1}, \alpha_{2}\right)$, where $\operatorname{Be}\left(\alpha_{1}, \alpha_{2}\right)=\int_{0}^{1} v^{\alpha_{1}-1}(1-v)^{\alpha_{2}-1} \mathrm{~d} v, \alpha_{1}, \alpha_{2}>0$, one gets

$$
\frac{1}{\Gamma(1-\alpha)} \int_{0}^{t}(t-u)^{-\alpha} u^{(\alpha+\beta) m-1} \mathrm{~d} u=\frac{t^{m(\beta+\alpha)-\alpha}}{\Gamma(1-\alpha)} \int_{0}^{1}(1-v)^{-\alpha} v^{m(\alpha+\beta)-1} \mathrm{~d} v=\frac{\Gamma(m(\alpha+\beta))}{\Gamma(m(\alpha+\beta)-\alpha+1)} t^{m(\beta+\alpha)-\alpha} .
$$

61

$$
\left({ }^{C} D_{0^{+}}^{\alpha} Y\right)(t)=\sum_{m=0}^{\infty} X_{m}(\alpha+\beta) m \frac{\Gamma(m(\alpha+\beta))}{\Gamma(m(\alpha+\beta)-\alpha+1)} t^{m(\beta+\alpha)-\alpha}=\sum_{m=0}^{\infty} X_{m+1} \frac{\Gamma((m+1)(\alpha+\beta)+1)}{\Gamma((m+1)(\alpha+\beta)-\alpha+1)} t^{(m+1)(\beta+\alpha)-\alpha}
$$

H1: For $m, m_{0}$ integers

$$
\exists \eta, \mathcal{H}>0, p \geq 0:\left\|B^{m}\right\|_{2} \leq \eta \mathcal{H}^{m-1}((m-1) !)^{p}, \quad \forall m: m \geq m_{0} \geq 1 .
$$

H2: $A$ and $B$ are independent RVs

Now,

$$
\left\|B^{m} A \prod_{n=1}^{m} \frac{\Gamma((n-1) \alpha+\beta n+1)}{\Gamma(n(\alpha+\beta)+1)} t^{(\alpha+\beta) m}\right\|_{2} \leq \eta \mathcal{H}^{m-1}((m-1) !)^{p}\|A\|_{2} \prod_{n=1}^{m} \frac{\Gamma((n-1) \alpha+\beta n+1)}{\Gamma(n(\alpha+\beta)+1)} t^{(\alpha+\beta) m}:=\delta_{m}(t) .
$$


The analysis of the convergence of the series $\sum_{m=0}^{\infty} \delta_{m}(t)$ will be performed by using the ratio or D'Alembert test. Indeed, we compute the $\lim _{m \rightarrow \infty} \frac{\delta_{m+1}(t)}{\delta_{m}(t)}$ with the aid of Stirling's formula, $\Gamma(x+1) \approx x^{x} e^{-x} \sqrt{2 \pi x}$ as $x \rightarrow \infty$ :

$$
\begin{aligned}
\lim _{m \rightarrow \infty} \frac{\delta_{m+1}(t)}{\delta_{m}(t)} & =\lim _{m \rightarrow \infty} \mathcal{H} m^{p} \frac{\Gamma(m \alpha+(m+1) \beta+1)}{\Gamma((m+1)(\alpha+\beta)+1)} t^{\alpha+\beta} \\
& =\lim _{m \rightarrow \infty} \frac{\mathcal{H} m^{p}(m \alpha+(m+1) \beta)^{m \alpha+(m+1) \beta} e^{-(m \alpha+(m+1) \beta)} \sqrt{2 \pi(m \alpha+(m+1) \beta)}}{[(m+1)(\alpha+\beta)]^{(m+1)(\alpha+\beta)} e^{-(m+1)(\alpha+\beta)} \sqrt{2 \pi(m+1)(\alpha+\beta)}} t^{\alpha+\beta} \\
& =\lim _{m \rightarrow \infty} \mathcal{H} m^{p}\left[\frac{m \alpha+(m+1) \beta}{(m+1) \beta+(m+1) \alpha}\right]^{m(\alpha+\beta)}\left[\frac{m \alpha+(m+1) \beta}{(m+1) \beta+(m+1) \alpha}\right]^{\beta} \\
& \times\left(\frac{1}{\beta+\alpha}\right)^{\alpha}\left(\frac{1}{m+1}\right)^{\alpha} e^{\alpha} \sqrt{\frac{m \alpha+(m+1) \beta}{(m+1) \beta+(m+1) \alpha}} t^{\alpha+\beta} .
\end{aligned}
$$

As $\lim _{m \rightarrow+\infty}\left[\frac{m \alpha+(m+1) \beta}{(m+1) \beta+(m+1) \alpha}\right]^{m}=e^{-\frac{\alpha}{\alpha+\beta}}, \lim _{m \rightarrow+\infty} \frac{m \alpha+(m+1) \beta}{(m+1) \beta+(m+1) \alpha}=1$, for $0 \leq p \leq \alpha$ and $t \geq 0$ it follows

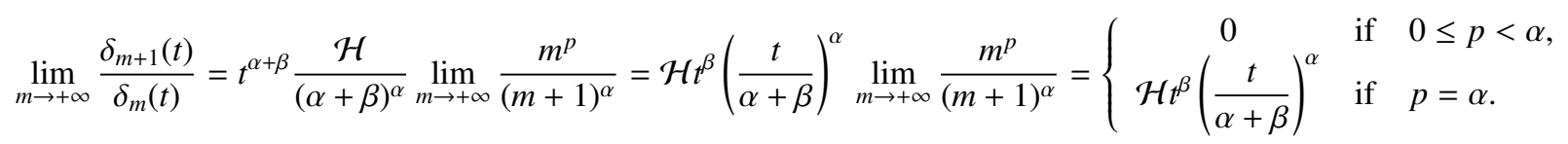

Therefore, the series $\sum_{m=0}^{\infty} \delta_{m}(t)$ is convergent for all $t$ in $\mathbb{D}$, where

$$
\mathbb{D}=\left\{\begin{array}{cll}
{[0, \infty[} & \text { if } & 0 \leq p<\alpha \\
{\left[0, \frac{(\alpha+\beta)^{\frac{\alpha}{\alpha+\beta}}}{\mathcal{H}^{\frac{1}{\alpha+\beta}}}\left[\begin{array}{ll}
\text { if } & p=\alpha
\end{array}\right.\right.}
\end{array}\right.
$$

which implies that $Y(t)$ is m.s. convergent for every $t$ in $\mathbb{D}$. Following a similar analysis, it can be shown that $Y^{\prime}(t)$ is m.s. convergent for every $t$ in $\mathbb{D}$. We conclude that for any closed interval $I$ in $\mathbb{D}, Y(t)$ is $\mathrm{m}$.s convergent on $I$ and $Y^{\prime}(t)$ m.s.u. convergent on $I$. As a consequence, hypotheses i) and ii) of Th. 2 hold. Hence, we have established the following result:

Theorem 3. If the RVs A, B satisfy conditions $H 1$ and H2, then the s.p. $Y(t)$ defined by (8) is a m.s. solution of the random IVP (1) on any closed interval $I \subset \mathbb{D}$, where $\mathbb{D}$ is defined in (11).

Remark 1. Although the random IVP (1) deals with the case that the order of the fractional derivative $\alpha$ lies in the interval $0<\alpha \leq 1$, it is worthy to point out that the ideas exhibited in this paper can be extended to the general scenario that $\alpha \in] n-1, n], n \geq 1$ integer. Just to illustrate the main ideas behind such extension, in the case that $n=2$, so $1<\alpha \leq 2$, then the solution stochastic process corresponding to RFDE given in (1) with random initial conditions $Y(0)=A_{1}$ and $Y^{\prime}(0)=A_{2}$, can be sought in the following form

$$
Y(t)=\hat{Y}_{0}(t)+\hat{Y}_{1}(t), \quad \hat{Y}_{0}(t)=\sum_{m=0}^{\infty} \hat{X}_{m, 0} t^{(\alpha+\beta) m}, \quad \hat{Y}_{1}(t)=\sum_{m=0}^{\infty} \hat{X}_{m, 1} t^{(\alpha+\beta) m+1} .
$$

Coefficients $\hat{X}_{m, 0}$ and $\hat{X}_{m, 1}$ can be determined via appropriate recurrences using the random Fröbenius technique.

Remark 2. It is important to remark that $\mathrm{H} 1$ is an implication of the quotient norm condition

$$
\exists p \geq 0: \frac{\left\|B^{m+1}\right\|_{2}}{\left\|B^{m}\right\|_{2}}=O\left(m^{p}\right), \quad \forall m: m \geq m_{0} \geq 1, \quad m, m_{0} \text { integers, }
$$

where $O(\cdot)$ denotes the Landau's symbol. By definition of $O(\cdot)$, condition (12) means

$$
\exists \mathcal{H}, p \geq 0:\left\|B^{m+1}\right\|_{2} \leq \mathcal{H} m^{p}\left\|B^{m}\right\|_{2}, \quad \forall m: m \geq m_{0} \geq 1, \quad m, m_{0} \text { integers. }
$$

By applying (13) recursively we obtain H1. Hence, the conclusion of Theorem 3 is valid if $B$ satisfies the quotient norm condition (12) and $A$ and $B$ satisfy H2. In some situations, the quotient condition is easier to check than $\mathrm{H} 1$. 
Remark 3. The set of RVs satisfying condition $\mathrm{H} 1$ is not empty. Important unbounded RVs satisfy condition H1. Indeed, for instance, a gaussian RVs, say $B$, with zero mean and finite variance, $\sigma^{2}<\infty, B \sim \mathrm{N}\left(0 ; \sigma^{2}\right)$ satisfies condition $\mathrm{H} 1$ for $p=1 / 2, \eta=\sigma>0$ and $\mathcal{H}=\sigma \sqrt{2}$ since

$$
\frac{\left\|B^{m+1}\right\|_{2}}{\left\|B^{m}\right\|_{2}}=\frac{\left(\mathbb{E}\left[B^{2(m+1)}\right]\right)^{1 / 2}}{\left(\mathbb{E}\left[B^{2 m}\right]\right)^{1 / 2}}=\sigma \sqrt{\frac{(2 m+2)(2 m+1)}{2(m+1)}}=O\left(m^{1 / 2}\right)
$$

where we have used that the moments w.r.t. the origin of $B$ are $\mathbb{E}\left[B^{2 n}\right]=\left(\sigma^{2 n}(2 n) !\right) /\left(2^{n} n !\right)$ (see [? ]). Additionally, it is straightforwardly to check that an important class of RVs satisfying condition H1 with $p=0$ are bounded RVs. As a consequence, significant RVs such that binomial, beta, uniform, triangular, etc. verify hypothesis $\mathrm{H} 1$. This fact is particularly useful from a practical standpoint since unbounded RVs can be approximated by truncating them so that the resulting bounded RV contains a prefixed mass of probability of the original unbounded RV.

As the m.s. solution $Y(t)$ of random IVP (1) is a 2-SP represented through an infinite series (see expression (8)), in practice, must be truncated at a positive integer $M$,

$$
Y_{M}(t)=A+\sum_{m=1}^{M} B^{m} A G_{m} t^{(\alpha+\beta) m}, \quad G_{m}:=\prod_{n=1}^{m} \frac{\Gamma((n-1) \alpha+\beta n+1)}{\Gamma(n(\alpha+\beta)+1)} .
$$

Its main relevant statistical information of $Y(t)$ is then given by the mean, the variance and the covariance functions of $Y_{M}(t)$. Considering that $A$ and $B$ are independent RVs, the mean of $Y_{M}(t)$ can be written as

$$
\mathbb{E}\left[Y_{M}(t)\right]=\mathbb{E}[A]+\sum_{m=1}^{M} \mathbb{E}\left[B^{m}\right] \mathbb{E}[A] G_{m} t^{(\alpha+\beta) m}
$$

As the covariance of two any $\operatorname{RVs} \tilde{A}$ and $\tilde{B}$ is defined by $\operatorname{Cov}[\tilde{A}, \tilde{B}]=\mathbb{E}[\tilde{A} \tilde{B}]-\mathbb{E}[\tilde{A}] \mathbb{E}[\tilde{B}]$, and in particular, $\operatorname{Cov}[\tilde{A}, \tilde{A}]=$ $\mathbb{E}\left[\tilde{A}^{2}\right]-(\mathbb{E}[\tilde{A}])^{2}=\mathbb{V}[\tilde{A}]$, where $\mathbb{V}[\tilde{A}]$ denotes the variance of $\tilde{A}$, the cross-covariance of $Y_{M}(t)$ and $Y_{N}(s)$ with $M, N$ positive integers and $t, s$ in $I \subset \mathbb{R}$ is given by

$$
\begin{aligned}
\operatorname{Cov}\left[Y_{M}(t), Y_{N}(s)\right] & =\mathbb{C o v}\left[A+\sum_{m=1}^{M} B^{m} A G_{m} t^{(\alpha+\beta) m}, A+\sum_{n=1}^{N} B^{n} A G_{n} s^{(\alpha+\beta) n}\right]+\sum_{n=1}^{N} \sum_{m=1}^{M} \mathbb{C o v}\left[B^{m} A, B^{n} A\right] G_{m} G_{n} t^{(\alpha+\beta) m} s^{(\alpha+\beta) n} \\
& =\mathbb{V}[A]\left(1+\sum_{n=1}^{N} \mathbb{E}\left[B^{n}\right] G_{n} t^{(\alpha+\beta) m}+\sum_{m=1}^{M} \mathbb{E}\left[B^{m}\right] G_{m} t^{(\alpha+\beta) m}\right) \\
& +\sum_{n=1}^{N} \sum_{m=1}^{M}\left(\mathbb{E}\left[A^{2}\right] \mathbb{E}\left[B^{m+n}\right]-(\mathbb{E}[A])^{2} \mathbb{E}\left[B^{m}\right] \mathbb{E}\left[B^{n}\right]\right) G_{m} G_{n} t^{(\alpha+\beta) m} s^{(\alpha+\beta) n}
\end{aligned}
$$

Since $\mathbb{V}\left[Y_{M}(t)\right]=\mathbb{C o v}\left[Y_{M}(t), Y_{M}(t)\right]$, setting $M=N$ and $t=s$ in (16), one also obtains the variance of $Y_{M}(t)$

$$
\mathbb{V}\left[Y_{M}(t)\right]=\mathbb{V}[A]\left(1+2 \sum_{m=1}^{M} \mathbb{E}\left[B^{m}\right] G_{m} t^{(\alpha+\beta) m}\right)+\sum_{n=1}^{M} \sum_{m=1}^{M}\left(\mathbb{E}\left[A^{2}\right] \mathbb{E}\left[B^{m+n}\right]-(\mathbb{E}[A])^{2} \mathbb{E}\left[B^{m}\right] \mathbb{E}\left[B^{n}\right]\right) G_{m} G_{n} t^{(\alpha+\beta)(m+n)}
$$

\section{Examples and Conclusions}

Now, we first illustrate the theoretical results previously established through two examples. The first one is a full numerical example while the second example illustrates the potentiality of random fractional IVP (1) in a mathematical modelling setting using real data. 
Example 1. This example has been devised to illustrate the different domains of convergence for the mean and standard deviation depending upon the relationship between parameters $p$ and $\alpha$ (see Th. 3 and expression (11)). Let us consider the random fractional IVP (1) in two scenarios (Cases I and II) depending on the order $\alpha \in] 0,1]$ of the fractional derivative, the parameter $\beta$ and the probability distributions chosen for $R V s B$ and $A$.

Case $1_{5}(p<\alpha): \alpha=0.7, B$ is a beta RV of parameters $(50,100)$, i.e., $B \sim B e(50 ; 100)$ (thus, according to Remark 3 , $p=0$

Case hh $h_{1}(p=\alpha): \alpha=0.5, B \sim N(0 ; 0.1)$ (thus, $\left.p=0.5\right), A \sim N(0.1 ; 1)$ and, $\beta=2$. As $p=\alpha$, according to Th. 3 and because $B$ is a bounded $R V) ; A$ is a Gaussian $R V$ with mean $\mu=0.1$ and variance $\sigma^{2}=1$, i.e., $A \sim N(0.1 ; 1)$ and, $\beta=0.1$. In Fig. 1, we have plotted approximations of the mean and standard deviation by expressions (15) and (17), respectively, using different orders of truncations $M$ over the interval $t \in[0,15]$. Notice that these results are in agreement with our theoretical findings. Indeed, as $p=0<0.7=\alpha$, we can observe that both statistical moments converge for every value of $t$.

expression (11), the domain of convergence is $\mathbb{D}=[0,2.626578[$ since $\eta=\sigma=0.1$ and $\mathcal{H}=\sigma \sqrt{2} \approx 0.141421$ (see Remark 3). In Fig. 2 , we have plotted approximations of the mean and standard deviation using different orders of truncations $M$ over the time intervals $t \in[0,3.5]$ and $t \in[0,3]$, respectively. To delineate the region of convergence we have plotted a vertical red line. For the sake of clarity, a part of the region of convergence has been magnified for both the mean and the standard deviation (right column of Case II in Fig. 2). The numerical results agree with theoretical findings.
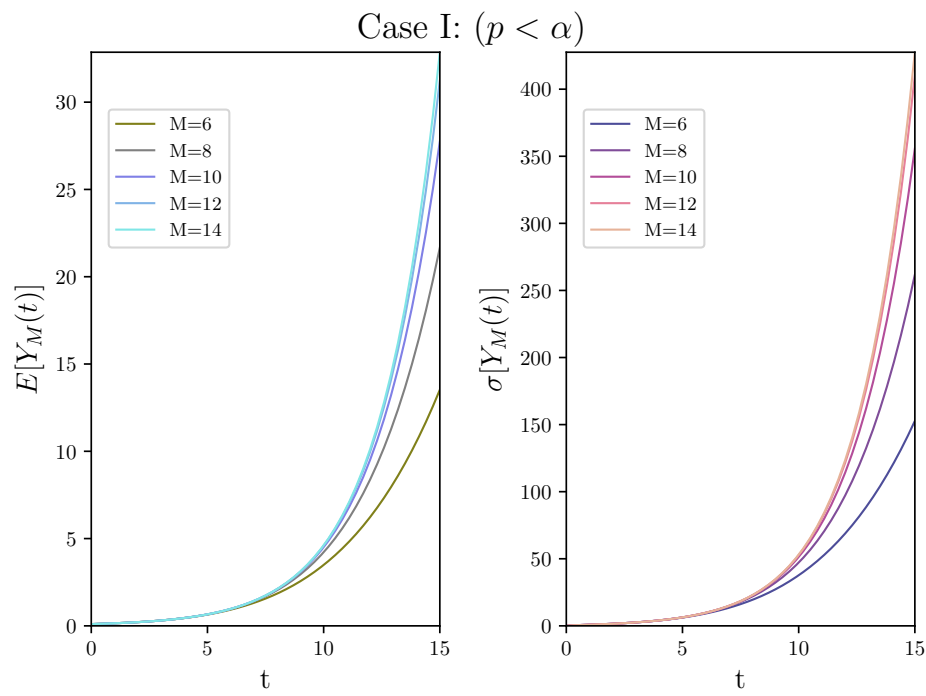

Figure 1: Approximations of the mean and the standard deviation of the solution SP to the random IVP (1) using different orders of truncations $M$ in Case I $(p<\alpha)$ described in the context of Example 1. Notice that the approximations corresponding to $M=12$ and $M=14$, for the mean and the standard deviation, match on the whole time interval $t \in[0,15]$, thus showing convergence.

When $p=\alpha$, as reported in (11), the domain of convergence $\left[0, t_{1}[\right.$ of the solution stochastic process may be small (it will depend on the fractional derivative order $\alpha, \beta$ model parameter and the constant $\mathcal{H}$ that appears in hypothesis H1). This domain $\left[0, t_{1}\right.$ [ with $t_{1}=\left((\alpha+\beta)^{\frac{\alpha}{\alpha+\beta}}\right) /\left(\mathcal{H}^{\frac{1}{\alpha+\beta}}\right)$, can be extended using the following strategy, which has been successfully applied in another contributions, [? ]. Once the solution $Y(t)$, given by $(8)$, has been constructed in the interval $\left[0, t_{1}\left[\right.\right.$, we seek a solution stochastic process, say $Y_{1}(t)$, of the form

$$
Y_{1}(t)=\sum_{m=0}^{\infty} X_{m, 1}\left(t-t_{1}\right)^{(\alpha+\beta) m},
$$

i.e., centered at $t_{1}$, of the same RFDE given in (1), but whose random initial condition matches the value of the solution, $Y(t)$, constructed in the piece $\left[0, t_{1}\right.$ [ at the ending time point, that is, $Y_{1}\left(t_{1}\right):=Y\left(t_{1}\right)$. Then, using a similar reasoning 

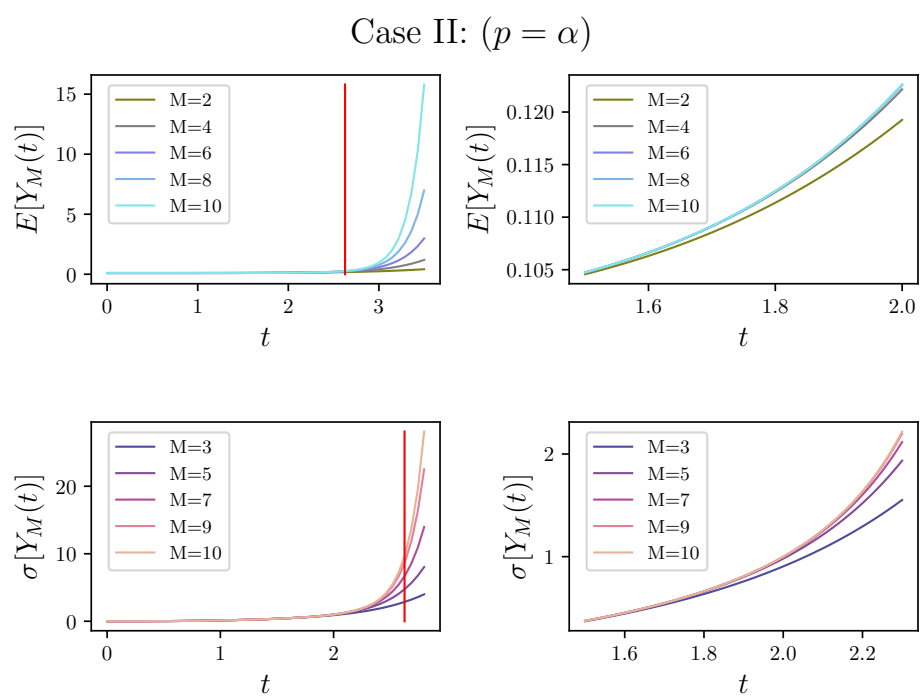

Figure 2: Approximations of the mean and the standard deviation of the solution SP to the random IVP (1) using different orders of truncations $M$ in Case II $(p=\alpha)$ described in the context of Example 1. On the left side, we have delineated the domain of convergence for the mean and the standard deviation plotting a vertical line. On the right side, we show a zoom on a piece of the domain of convergence, for the sake of clarity. Observe that the approximations corresponding to $M=4,6,8,10$ match for the mean, while this same fact happens when $M=9,10$, in the case of the standard deviation.

we have exhibited in our development, one can determine the new coefficient random variables $X_{m, 1}, m \geq 0$, and it can be proven that random series (18) is m.s. convergent in the piece $\left[t_{1}, 2 t_{1}\right.$ [. This procedure can be successively applied to extend the solution on a desired interval, say $[0, T]$.

Example 2. Now, we illustrate an application of random fractional IVP (1) to model the dynamics of growth bacteria over the time using real data. Differential equation in (1) can be interpreted as a generalization of the classical exponential (or Malthusian) model with time-dependent population growth rate, Bt ${ }^{\beta}$, for a species whose initial population, A is known. Here, this generalization has been made in two senses, namely, first introducing the Caputo fractional derivative, $\left({ }^{C} D_{0^{+}}^{\alpha} Y\right)(t)$ with $0<\alpha \leq 1$, instead of classical derivative, $Y^{\prime}(t)$, and secondly, by considering model parameter $B$ and initial condition $A$ as RVs rather than deterministic values. On the one hand, the use of a fractional derivative can be justified because the growth dynamics is determined by genetic, environmental factors, etc., developed over previous periods, then it is expected these biological features can be better modelled via Caputo fractional derivative, which is defined in terms of an integral (thus with memory), instead of classical derivative that just characterizes instantaneous changes. On the other hand, the consideration of randomness in model inputs $B$ and A can be justified because the complex nature of population growth rate, which depends on uncertain biological factors, and, in practice, the value of the initial condition is usually known on the basis of sampling, respectively. In this spirit, here we consider the classical non-autonomous Malthus model to a generalized one, in which ordinary derivative and model inputs are replaced by fractional derivatives and RVs, respectively. Our example is based on measured population values of Rhodobacter Capsulatus anaerobic photosynthetic bacteria $\left\{y_{i}: 0 \leq i \leq 4\right\}$ corresponding to days $t_{i}$ (Table 1). This information has been obtained from source [? ]. First, we have performed a classical (or deterministic) fitting based upon minimizing the mean square error between real data $y_{i}$, and the solution of the corresponding deterministic differential equation $\left(Y^{\prime}(t)=B t^{\beta} Y(t), Y(0)=A=5.83 \times 10^{5}\right)$, which is given by

$$
Y(t)=A \exp \left(\frac{B}{1+\beta}+\frac{B t^{1+\beta}}{1+\beta}\right) .
$$

Using PSO (Particle Swarm Optimization method) with 1000 iterations [?], we have obtained the following estimates for deterministic model parameters: $\beta=2.2573$ and $B=0.168764$, being the RMSE (Root Mean Square Error) of this fitting $\epsilon^{\text {det. }}=763$ (observe that units are of magnitude $10^{6}$. In Table 1 we show the results, $y_{i}^{\text {det. }}$, provided by this 
approach. Secondly, we have assumed that $B$ is a Gamma $R V$ of parameters $\left(r_{B}, s_{B}\right)$ and the initial condition $A$ is an Exponential $R V$ of parameter $\lambda_{A}=1 /\left(5.83 \times 10^{5}\right)$. On the one hand, observe that the choice made for the distribution of $B$ is justified because real data $y_{i}$ have a positive trend, hence $B$ must be a positive $R V$ and Gamma distribution holds this feature, moreover it is a flexible distribution able to perform a good fitting since it depends on two parameters $\left(r_{B}\right.$ and $\left.s_{B}\right)$. On the other hand, Exponential distribution guarantees the positiveness of the initial condition, and we have imposed that its mean $\mathbb{E}[A]=1 / \lambda_{A}$ matches the initial condition $5.83 \times 10^{5}$. Then, considering this choice for the distributions of random inputs $B$ and $A$, we have performed a (random) fitting based upon minimizing the mean square error between real data $y_{i}$ and the mean of the solution stochastic process of the random fractional IVP (1), i.e.,

$$
\min _{r_{B}, s_{B}, \beta>0 ; 0<\alpha \leq 1} \operatorname{Error}\left(r_{B}, s_{B}, \beta, \alpha\right)=\sum_{i=0}^{4}\left(y_{i}-\mathbb{E}\left[Y_{M}\left(t_{i} ; r_{B}, s_{B}, \beta, \alpha\right)\right]\right)^{2},
$$

where $\mathbb{E}\left[Y_{M}\left(t_{i} ; r_{B}, s_{B}, \beta, \alpha\right)\right]$ is given by (14)-(15). We have again applied PSO method with 1000 iterations to solve this minimization program taking as truncation order $M=20$ (for which the approximation of the exact expectation is very accurate) and then we have obtained the following estimates for model parameters: $\beta=0.1975, r_{B}=14.64, s_{B}=$ 75.32 and $\alpha=0.89$, being the RMSE (Root Mean Square Error) of this fitting $\epsilon^{\text {random }}=660$. In Table 1 we show the results, $y_{i}^{\text {random }}$, provided by this approach. We have shown that $\epsilon^{\text {random }}<\epsilon^{\text {det. }}$, in order to complete better an adequate comparison between deterministic and random fractional approaches, in Table 1 we give an important goodness-offit measure, MAPE (Mean Absolute Percentage Error). Again, we can observe that our proposed approach provides better results for this statistical measure.

\begin{tabular}{|c|c|c|c|c|c|c|}
\hline$t_{i}$ (time in days) & 0 & 2 & 4 & 7 & 9 & MAPE \\
\hline$y_{i}$ (population cells $\left./ \mathrm{mL}\right)$ & $5.830 \mathrm{E}+05$ & $6.350 \mathrm{E}+05$ & $1.08 \mathrm{E}+06$ & $3.20 \mathrm{E}+06$ & $5.23 \mathrm{E}+06$ & - \\
\hline \hline$y_{i}^{\text {det. }}($ deterministic fitting) & $6.667 \mathrm{E}+05$ & $9.189 \mathrm{E}+05$ & $1.435 \mathrm{E}+06$ & $3.141 \mathrm{E}+06$ & $5.589 \mathrm{E}+06$ & 0.20144 \\
\hline$y_{i}^{\text {random }}$ (random fitting) & $5.830 \mathrm{E}+05$ & $8.504 \mathrm{E}+05$ & $1.338 \mathrm{E}+06$ & $2.932 \mathrm{E}+06$ & $5.307 \mathrm{E}+06$ & 0.13533 \\
\hline
\end{tabular}

Table 1: Cell counts $y_{i}$ of Rhodobacter Capsulatus anaerobic photosynthetic bacteria at the time instants $t_{i}$ (data retrieved from [? ]). Values of the determinsitic fitting $\left(y_{i}^{\text {det. }}\right)$ and random fractional fitting $y_{i}^{\text {random }}$. Goodness-of-fit measure for both approaches: MAPE (Mean Absolute Percentage Error). Example 2.

Finally, we want to underline that this study seeks to contribute to the emergent area of random fractional differential equations (RFDEs) where the areas of fractional calculus and differential equations meet to provide a rigorous treatment of randomness. We think that the generality of fractional derivatives and the powerful of differential equations will give RFDEs a prominent role also in modelling phenomena with uncertainty.

\section{Acknowledgements}

Authors gratefully acknowledge the comments made by reviewers, which have greatly enriched the manuscript. This work has been partially supported by Ministerio de Economía y Competitividad grant MTM2013-41765-P.

\section{Conflict of Interest Statement}

The authors declare that there is no conflict of interests regarding the publication of this article. 